\title{
ESTABILIDADE ROBUSTA DE SISTEMAS NEUTRAIS COM ATRASOS VARIANTES NO TEMPO
}

\author{
Valter Júnior de Souza Leite* \\ valter@ieee.org \\ Eugênio B. Castelan \\ eugenioddas.ufsc.br
}

\author{
Pedro Luis Dias Peres ${ }^{\dagger}$ \\ peres@dt.fee.unicamp.br
}

Sophie Tarbouriech ${ }^{\S}$

tarbour@laas.fr

\author{
${ }^{*}$ UnED Divinópolis - CEFET-MG \\ R. Monte Santo, 319, CEP 35502-036 \\ Divinópolis, MG, Brasil \\ ${ }^{\dagger} \mathrm{DT} / \mathrm{FEEC} / \mathrm{UNICAMP}$ \\ CP 6101, CEP 13083-970 \\ Campinas, SP, Brasil \\ ${ }^{\ddagger}$ DAS / CTC / UFSC \\ CEP 88040-900 \\ Florianópolis - SC - Brasil \\ ${ }^{\S}$ LAAS-CNRS, University of Toulouse, \\ 7, Avenue du Colonel Roche, 31077, CEDEX 4 \\ Toulouse, França
}

\begin{abstract}
Sufficient linear matrix inequality conditions are given for robust stability analysis of linear uncertain neutral systems, where it is assumed that the vector of states has timevarying delays. All system matrices are supposed to be time invariant, uncertain but belonging to a polytope with known vertices. The robust stability of the uncertain neutral system is assured by means of a parameter dependent LyapunovKrasovskii functional. Additionally, it is shown how robust stability conditions for uncertain continuous-time systems with and without state delays can be recovered from the conditions proposed in the paper. Numerical examples illustrate the obtained results.
\end{abstract}

Artigo submetido em 14/04/2005

1a. Revisão em 22/05/2006

2a. Revisão em 04/07/2007

Aceito sob recomendação do Editor Associado Prof. Liu Hsu
KEYWORDS: Robust stability, neutral systems, timevarying delays, parameter dependent Lyapunov-Krasovskii functional.

\section{RESUMO}

Condições suficientes na forma de desigualdades matriciais lineares são dadas para a análise de estabilidade robusta de sistemas lineares incertos do tipo neutral, nos quais assumese que o vetor de estados possui atrasos variantes no tempo. Todas as matrizes do sistema são supostas invariantes no tempo, incertas porém pertencentes a um politopo cujos vértices são conhecidos. A estabilidade robusta do sistema incerto neutral é assegurada por meio de um funcional de Lyapunov-Krasovskii dependente de parâmetro. Além disso, mostra-se como condições para a estabilidade robusta de sistemas incertos contínuos no tempo com e sem atraso nos estados podem ser recuperadas a partir das condições 
propostas no trabalho. Exemplos numéricos ilustram os resultados obtidos.

PALAVRAS-CHAVE: Estabilidade robusta, sistemas neutrais, atrasos variantes no tempo, funcional de LyapunovKrasovskii dependente de parâmetro.

\section{INTRODUÇÃO}

A classe dos sistemas lineares com atraso nos estados tem sido um importante objeto de estudo nos últimos anos. O interesse nessa classe de sistemas é justificado principalmente pelos efeitos negativos que os atrasos exercem sobre a estabilidade dos sistemas. Além disso, pode-se dizer que a presença de atrasos em sistemas reais é uma característica freqüente. Veja, por exemplo, Gu et al. (2003), Gu e Niculescu (2003), Richard (2003), Niculescu (2001), Mahmoud (2000), Kolmanovskii e Richard (1999), Dugard e Verriest (1997), Hale e Lunel (1993), MalekZavarei e Jamshidi (1987) e referências internas para um apanhado de exemplos, técnicas de análise e de síntese de controladores para sistemas com atraso. A presença de incertezas nos parâmetros do sistemas torna a tarefa de assegurar a estabilidade robusta ainda mais complexa. Assim, uma das principais abordagens utilizadaspara a análise de estabilidade desse tipo de sistema é, sem dúvida, o uso de funcionais de Lyapunov-Krasovskii (Kolmanovskii et al., 1999), (Skorodinskii, 1990), aliada a um esforço significativo para a caracterização da estabilidade robusta por meio de desigualdades matriciais lineares (LMIs, do inglês Linear Matrix Inequalities).

Existem dois tipos de condições para a análise de estabilidade para a classe de sistemas lineares com atraso nos estados: as que são dependentes do atraso e as que são independentes do atraso. No primeiro caso as condições visam garantir a estabilidade de um sistema com atraso nos estados para todo valor do atraso $\tau \in[0, \bar{\tau}]$, e $\bar{\tau}$ denota o máximo valor assumido por $\tau$. As condições independentes do atraso são capazes de garantir (quando satisfeitas) a estabilidade de sistemas com atraso para qualquer valor (positivo) de $\tau$. Como, em geral, as condições dependentes do atraso são apenas suficientes, nem sempre é possível utilizar condições dependentes do atraso para caracterizar adequadamente a estabilidade de um sistema que seja estável independentemente do atraso.

A classe de sistemas cuja dinâmica depende da derivada dos estados passados é descrita por equações diferenciais hiperbólicas cuja formalização contém as equações diferenciais ordinárias normalmente utilizadas no contexto da teoria de controle (Hale e Lunel, 1993). Linhas de transmissão (veja Bellen et al. (1999)), modelos equivalentes com elementos parciais (PEEC, do inglês Partial Element
Equivalent Circuit), utilizados na modelagem de sistemas eletrônicos complexos (veja por exemplo Cullum et al. (2000) e Yue e Han (2004)), circuitos que operam em alta freqüência, problemas de propagação de ondas eletromagnéticas tridimensionais em circuitos, e alguns processos dinâmicos que ocorrem em tubulações de vapor ou fluido, são exemplos de sistemas físicos que podem ser modelados através de equações diferenciais hiperbólicas com condições iniciais adequadas e derivadas como condições de contorno. Detalhes sobre esses (e outros) sistemas neutrais podem ser encontrados em Hale e Lunel (1993), Niculescu (2001), Cullum et al. (2000) e Bellen et al. (1999), Kolmanovskii e Myshkis (1998).

Dentre diversos trabalhos tratando dessa classe de sistemas, pode-se citar Niculescu (2001) em que são formuladas tanto condições dependentes do atraso quanto condições independentes do atraso para sistemas conhecidos e invariantes no tempo. Condições dependentes do atraso também são dadas em Bin et al. (2003) para esse mesmo tipo de sistema (conhecido e invariante no tempo). Sistemas variantes no tempo e com atrasos também variantes no tempo são tratados em Verriest (1999) através de condições independentes do atraso, formuladas em termos de equações algébricas de Riccati. Incertezas do tipo limitada em norma são consideradas em Xu et al. (2003) para sistemas invariantes no tempo e atraso fixo. Bliman (2002) propõe condições suficientes independentes do atraso, que tendem à necessidade na medida em que a complexidade das inequações matriciais é aumentada, são propostas. No sistema neutral

$$
\dot{x}(t)-E \dot{x}(t-\tau)=A(\alpha) x(t)+A_{h}(\alpha) x(t-h)
$$

investigado em Bliman (2002), assume-se a matriz $E$ precisamente conhecida, os atrasos $\tau$ e $h$ iguais $\mathrm{e}$ invariantes no tempo e as matrizes dinâmicas $A(\alpha)$ e $A_{h}(\alpha)$ pertencentes a um politopo de vértices conhecidos. Uma abordagem que utiliza a teoria de sistemas descritores foi introduzida em Fridman (2001). Esse trabalho não considera incertezas no modelo nem parâmetros variantes no tempo. Outros resultados relevantes incluem Park (2003), Cao e He (2004), Chen (2003), Park et al. (2004), Chen (2004), Fu (2004), Park e Won (2000), Park (2001), Han (2002), Han (2004), Ivănescu et al. (2003). No entanto, a maior parte desses trabalhos considera apenas atrasos invariantes no tempo e, via de regra, assume que o mesmo atraso afeta o estado e sua derivada, isto é, $h=\tau$. Embora na maioria dos sistemas modelados por equações neutrais os atrasos $h$ e $\tau$ sejam considerados idênticos, em alguns livros aparecem modelos com atrasos distintos que propiciam uma descrição mais abrangente (Kolmanovskii e Myshkis, 1998), (Kolmanovskii e Myshkis, 1992). Neste trabalho, optou-se por manter $h$ e $\tau$ possivelmente distintos, descrevendo uma situação mais geral que contém o caso 
$h=\tau$. Em geral, a estabilidade quadrática é utilizada na maior parte dos resultados citados anteriormente. No contexto de sistemas com atraso sem o termo neutral (i.e. $E=\mathbf{0}$ ), é importante citar Zhang et al. (2001) em que é demonstrada, através do uso do lema do ganho pequeno com matrizes constantes de escalonamento, a equivalência de várias condições formuladas através de funcionais de Lyapunov-Krasovskii e a análise de estabilidade robusta de um sistema de comparação livre de atrasos. Não são, no entanto, admitidas incertezas no sistema original e o atraso é assumido constante. Em Kao e Rantzer (2003) é tratada a estabilidade de sistemas lineares com matrizes conhecidas e atrasos variantes no tempo. Nesse último, é utilizada uma formulação baseada em restrições de integrais quadráticas (IQCs, do inglês Integral Quadratic Constraints). Note ainda que, conforme argumentado em Kao e Rantzer (2003), no contexto de sistemas com atraso $(E=\mathbf{0})$, quando o atraso é variante no tempo a obtenção de condições para a análise de estabilidade torna-se mais elaborada.

A abordagem da estabilidade quadrática (Barmish, 1985) é freqüentemente utilizada para tratar a presença de incertezas, porém apresentando muitas vezes um elevado grau de conservadorismo. Assim, condições menos conservadoras têm sido obtidas através do uso de funções de Lyapunov dependentes de parâmetro no contexto da estabilidade robusta de sistemas lineares incertos (de Oliveira et al., 1999), (Leite e Peres, 2003), (Peaucelle et al., 2000), (Ramos e Peres, 2001), (Ramos e Peres, 2002), (Leite et al., 2004).

Neste trabalho, funcionais dependentes de parâmetro são utilizados para o estudo da análise de estabilidade robusta de sistemas neutrais incertos com atrasos variantes no tempo. São desenvolvidas condições do tipo independente do atraso para sistemas incertos. O principal objetivo aqui é obter condições convexas no formato de LMIs para o estudo da estabilidade robusta de sistemas incertos do tipo neutral, ou seja, para a classe de sistemas cuja dinâmica depende da derivada dos estados passados. Diferentemente da maioria dos trabalhos que tratam desse assunto, valores distintos de atraso de tempo são considerados nos estados atrasados e em suas derivadas. Além disso, nenhuma transformação de modelo, que normalmente introduz novas dinâmicas no modelo construído, conforme discutido em Gu e Niculescu (2001), (Niculescu, 2001) é utilizada e todas as matrizes de sistema podem ser afetadas por incertezas do tipo politópica com vértices conhecidos. Condições suficientes na forma de LMIs são propostas para a existência de um funcional de Lyapunov-Krasovskii dependente de parâmetro, assegurando a estabilidade robusta do sistema neutral incerto independentemente dos valores dos atrasos variantes no tempo. Embora apresentadas para o caso de atrasos simples, as condições podem ser facilmente estendidas para o caso de múltiplos atrasos. É mostrado que a formulação apresentada neste trabalho contém outros resultados da literatura, tais como a estabilidade robusta de sistemas lineares incertos livres de atrasos e a estabilidade robusta de sistemas incertos com atrasos, como casos especiais. Exemplos numéricos ilustram a efetividade dos resultados.

\subsection{Notação}

Neste texto, $M^{\prime}$ indica a transposta de $M, M<0(\leq 0)$ significa que $M$ é simétrica (semi-) definida negativa. $N$ é usado para denotar o número de vértices de um politopo. $\mathbb{R}$ é o conjunto dos números reais, $\mathbb{R}_{+}$o conjunto dos números reais positivos, $\mathbb{R}^{n}$ o espaço dos vetores com $n$ elementos reais e $\mathbb{R}^{n \times n}$ é o espaço das matrizes de dimensões $n \times n$ com entradas reais. $\mathbf{I}_{n}$ denota a matriz identidade de dimensões $n \times n$ e $\mathbf{0}$ é utilizado para denotar a matriz nula de dimensões apropriadas. $\quad \mathcal{C}_{\tau}=\mathcal{C}\left([-\tau, 0], \mathbb{R}^{n}\right)$ denota o espaço de Banach das funções vetoriais contínuas mapeando o intervalo $[-\tau, 0]$ em $\mathbb{R}^{n}$ com topologia de convergência uniforme. $\mathcal{C}_{\tau}^{v}$ é o conjunto definido como $\mathcal{C}_{\tau}^{v}=\left\{\phi \in \mathcal{C}_{\tau} ;\|\phi\|_{c}<v, v>\right.$ $0\}$, em que $\|\phi\|_{c}=\sup _{-\tau \leq t \leq 0}\|\phi(t)\|$, sendo $\|(\cdot)\|$ a norma 2 de $(\cdot)$. O símbolo $\star$ representa os blocos simétricos nas LMIs.

\section{PRELIMINARES}

Considere a seguinte classe de sistemas incertos neutrais

$$
\begin{gathered}
\frac{\partial}{\partial t} \boldsymbol{\Delta}\left(x_{\tau}\right)=A(\alpha) x(t)+A_{h}(\alpha) x(t-h(t)) \\
\Delta\left(x_{\tau}\right) \triangleq x(t)-E(\alpha) x(t-\tau(t))
\end{gathered}
$$

com condições iniciais

$$
\begin{array}{r}
x\left(t_{0}+\xi\right)=\phi(\xi), \forall \xi \in[-\varsigma, 0],\left(t_{0}, \phi\right) \in \mathbb{R}^{+} \times \mathcal{C}_{\varsigma}^{v} \\
\varsigma \triangleq \max \{h(t), \tau(t))\}, h(t) \geq 0, \tau(t) \geq 0, \forall t
\end{array}
$$

em que $x(t) \in \mathbb{R}^{n}$ é o vetor de estados e $h(t) \in \mathbb{R}_{+}$e $\tau(t) \in$ $\mathbb{R}_{+}$são atrasos variantes no tempo. As matrizes invariantes no tempo $E(\alpha), A(\alpha)$ e $A_{h}(\alpha)$ não são precisamente conhecidas, mas pertencem a um domínio politópico $\mathcal{P}$ com vértices conhecidos $E_{j}, A_{j}, A_{h j}-$ ou $\left(E, A, A_{h}\right)_{j}$ - dado por

$$
\begin{gathered}
\mathcal{P}=\left\{\left(E, A, A_{h}\right)(\alpha) \in \mathbb{R}^{n \times 3 n}:\right. \\
\left(E, A, A_{h}\right)(\alpha)=\sum_{j=1}^{N} \alpha_{j}\left(E, A, A_{h}\right)_{j} ; \\
\left.\sum_{j=1}^{N} \alpha_{j}=1 ; \alpha_{j} \geq 0\right\}
\end{gathered}
$$

Portanto, qualquer tripla $\left(E, A, A_{h}\right)(\alpha)$ em $\mathcal{P}$ pode ser escrita como uma combinação convexa dos vértices 
$\left(E, A, A_{h}\right)_{j}$ do politopo de incertezas, em termos de $\alpha$, $\alpha_{j} \geq 0, \sum_{j=1}^{N} \alpha_{j}=1$.

Neste trabalho as seguintes definições são adotadas:

Definição 1 É chamado sistema linear incerto neutral todo aquele que pode ser modelado conforme (1), $\operatorname{com} E(\alpha) \neq \mathbf{0}$ para todo $\alpha$ admissível.

Definição 2 É chamado sistema linear incerto com atraso todo aquele que pode ser modelado conforme (1), com $E(\alpha)=\mathbf{0}$ para todo $\alpha$ admissível e $A_{h}(\alpha) \neq \mathbf{0}$ para algum $\alpha$.

Neste trabalho, o operador $\boldsymbol{\Delta}(\cdot)$, definido em (2), é suposto uniformemente estável ( $\mathrm{Gu}$ et al., 2003). No caso em que $\tau(t)$ é invariante no tempo, isto é, $\tau(t)=\tau$, então $\boldsymbol{\Delta}(\cdot)$ é uniformemente estável se

$$
\rho(E(\alpha))<1, \forall \alpha \text { admissível. }
$$

Essa condição é conhecida na literatura como a estabilidade Schur-Cohn de $E(\alpha)$ (Vieira e Kailath, 1977), (Niculescu, 2001).

Entretanto, no caso mais geral em que $\tau(t)$ é variante no tempo, as condições que asseguram a estabilidade uniforme de $\boldsymbol{\Delta}(\cdot)$ não são bem estabelecidas na literatura. Para mais detalhes no caso de sistemas com atrasos variantes no tempo, veja El'sgol'ts (1966).

O problema a ser investigado é:

Problema 1 Determine, se possível, condições que assegurem a estabilidade robusta para o sistema linear incerto neutral (1)-(5) independentemente dos valores dos atrasos variantes no tempo $h(t)$ e $\tau(t)$.

Condições suficientes para resolver o Problema 1 são dadas em termos de LMIs. Para isso, um funcional de Lyapunov-Krasovskii dependente de parâmetro é usado, proporcionando condições convexas do tipo "independente do atraso" que são menos conservadoras que outras similares obtidas através da abordagem de estabilidade quadrática, isto é, utilizando matrizes de Lyapunov-Krasovskii constantes e independentes de parâmetro.

\section{ESTABILIDADE INDEPENDENTE DO ATRASO}

Inicialmente, uma LMI de dimensão infinita em $\alpha$ é dada como solução do Problema 1. A partir dessa condição, uma outra é proposta, suficiente para que a primeira seja verificada, desta vez definida em um número finito de LMIs.
Para isso, algumas variáveis matriciais são escolhidas como independentes do parâmetro $\alpha$.

O lema seguinte, oriundo da teoria de estabilidade de Lyapunov, é usado para a obtenção dos resultados deste trabalho. Para detalhes, veja, por exemplo, (Niculescu, 2001), (Gu et al., 2003), (Hale, 1977), (Hale e Lunel, 1993).

Lema 1 O sistema linear incerto neutral (1)-(5) é robustamente estável independentemente dos valores dos atrasos variantes no tempo $h(t)$ e $\tau(t)$ se existirem matrizes simétricas definidas positivas $P(\alpha) \in \mathbb{R}^{n \times n}$, $S_{1}(\alpha) \in \mathbb{R}^{n \times n}$ e $S_{2}(\alpha) \in \mathbb{R}^{n \times n}$ tais que

$$
\begin{aligned}
& V(\alpha, x(t), h(t), \tau(t))=V_{1}\left(\alpha, \Delta\left(x_{\tau}\right)\right) \\
& +V_{2}(\alpha, x(t), h(t))+V_{3}(\alpha, x(t), \tau(t))>0
\end{aligned}
$$

em que

$$
\begin{gathered}
V_{1}\left(\alpha, \boldsymbol{\Delta}\left(x_{\tau}\right)\right) \triangleq \Delta\left(x_{\tau}\right)^{\prime} P(\alpha) \Delta\left(x_{\tau}\right) \\
V_{2}(\alpha, x(t), h(t)) \triangleq \int_{t-h(t)}^{t} x(\sigma)^{\prime} S_{1}(\alpha) x(\sigma) d \sigma \\
V_{3}(\alpha, x(t), \tau(t)) \triangleq \int_{t-\tau(t)}^{t} x(\varepsilon)^{\prime} S_{2}(\alpha) x(\varepsilon) d \varepsilon
\end{gathered}
$$

para todo $\chi(t) \neq \mathbf{0}$

$$
\chi(t)=\left[\begin{array}{c}
x(t) \\
x(t-h(t)) \\
x(t-\tau(t))
\end{array}\right]
$$

$$
\frac{\partial}{\partial t} V(\alpha, x(t), h(t), \tau(t))<0
$$

Prova: As condições (6) e (11) são suficientes para a estabilidade assintótica do sistema linear incerto neutral (1)(5), com o operador $\boldsymbol{\Delta}(\cdot)$ uniformemente estável, conforme mostrado, por exemplo, em Niculescu (2001).

Note que a condição suficiente do Lema 1 para a estabilidade assintótica robusta exclui o chamado caso crítico de sistemas neutrais (cadeia de raízes tendendo ao eixo imaginário). O Lema 1 pode ser visto como uma aplicação do chamado Teorema de Lyapunov-Krasovskii (Gu et al., 2003, Theorem 3.1) ao caso de sistemas neutrais com incerteza politópica. Estratégia semelhante foi apresentada em Bliman (2002) para tratar sistemas neutrais com matriz $E(\alpha)=E$ precisamente conhecida e atrasos iguais invariantes no tempo $\tau=h$, fazendo apelo a resultados de (Hale, 1977).

Uma observação importante a respeito do Lema 1 é que esse lema não proporciona um método para a obtenção das 
matrizes $P(\alpha), S_{1}(\alpha)$ e $S_{2}(\alpha)$. Note ainda que a equação (11) é dada por

$$
\begin{aligned}
\frac{\partial}{\partial t} V(\alpha, x(t), h(t), \tau(t)) \\
=\dot{\boldsymbol{\Delta}}\left(x_{\tau}\right)^{\prime} P(\alpha) \boldsymbol{\Delta}\left(x_{\tau}\right)+\boldsymbol{\Delta}\left(x_{\tau}\right)^{\prime} P(\alpha) \dot{\boldsymbol{\Delta}}\left(x_{\tau}\right) \\
-(1-\dot{h}(t)) x(t-h(t))^{\prime} S_{1}(\alpha) x(t-h(t)) \\
-(1-\dot{\tau}(t)) x(t-\tau(t))^{\prime} S_{2}(\alpha) x(t-\tau(t)) \\
\quad+x(t)^{\prime}\left(S_{1}(\alpha)+S_{2}(\alpha)\right) x(t)<\mathbf{0}
\end{aligned}
$$

em que nenhum majorante sobre as taxas de variação dos atrasos é imposto. Deste ponto em diante, os seguintes limites são assumidos para as taxas de variação dos atrasos, como forma de obter condições suficientes para a verificação do Lema 1 e, portanto, fornecer uma solução para o Problema 1. Assim, suponha que

$$
\begin{aligned}
& |\dot{h}(t)| \leq \bar{h}<1 \\
& |\dot{\tau}(t)| \leq \bar{\tau}<1
\end{aligned}
$$

e defina os seguintes escalares

$$
\beta_{\bar{h}} \triangleq(1-\bar{h}) ; \beta_{\bar{\tau}} \triangleq(1-\bar{\tau})
$$

Com essas definições é possível estabelecer os principais resultados deste trabalho.

Teorema 1 As condições equivalentes seguintes garantem a estabilidade robusta independentemente dos atrasos variantes no tempo do sistema linear incerto neutral (1)-(5), sujeitos a (13)-(14), e são suficientes para a verificação do Lema 1, assegurando portanto, uma solução para o Problema 1:

i) Existem matrizes simétricas definidas positivas $P(\alpha) \in$ $\mathbb{R}^{n \times n}, S_{1}(\alpha) \in \mathbb{R}^{n \times n}, S_{2}(\alpha) \in \mathbb{R}^{n \times n}$ tais que

$$
\Theta(\alpha) \triangleq\left[\begin{array}{cc}
A(\alpha)^{\prime} P(\alpha)+P(\alpha) A(\alpha) & P(\alpha) A_{h}(\alpha) \\
+S_{1}(\alpha)+S_{2}(\alpha) & -\beta_{\bar{h}} S_{1}(\alpha) \\
\star & \star \\
\star & \\
-A(\alpha)^{\prime} P(\alpha) E(\alpha) \\
-A_{h}(\alpha)^{\prime} P(\alpha) E(\alpha) \\
-\beta_{\bar{\tau}} S_{2}(\alpha)
\end{array}\right]<\mathbf{0} ; \forall \alpha
$$

ii) Existem matrizes simétricas definidas positivas $P(\alpha) \in$ $\mathbb{R}^{n \times n}, \quad S_{1}(\alpha) \in \mathbb{R}^{n \times n}, S_{2}(\alpha) \in \mathbb{R}^{n \times n}$ e matrizes $F_{1}(\alpha), F_{2}(\alpha), G_{1}(\alpha), G_{2}(\alpha), H_{1}(\alpha), H_{2}(\alpha), M_{1}(\alpha)$, $M_{2}(\alpha), N_{1}(\alpha)$ e $N_{2}(\alpha)$ pertencentes a $\mathbb{R}^{n \times n}$ tais que (17) é verificada para todo $\alpha$ admissível.

Prova: O fato de que i) é uma condição suficiente para resolver o Problema 1 vem diretamente do Lema 1.
Avaliando a derivada temporal de $V(\cdot)$, com $\boldsymbol{\Delta}\left(x_{\tau}\right)$ dado em (2), obtém-se $\chi(t)^{\prime} \tilde{\Theta}(\alpha, t) \chi(t), \operatorname{com} \chi(t)$ dado em (10) e

$$
\begin{aligned}
& \tilde{\Theta}(\alpha, t) \triangleq \\
& {\left[\begin{array}{cc}
A(\alpha)^{\prime} P(\alpha)+P(\alpha) A(\alpha) & P(\alpha) A_{h}(\alpha) \\
+S_{1}(\alpha)+S_{2}(\alpha) & -\beta_{\dot{h}} S_{1}(\alpha) \\
\star & \star
\end{array}\right.} \\
& \left.\begin{array}{c}
-A(\alpha)^{\prime} P(\alpha) E(\alpha) \\
-A_{h}(\alpha)^{\prime} P(\alpha) E(\alpha) \\
-\beta_{\dot{\tau}} S_{2}(\alpha)
\end{array}\right]<\mathbf{0}
\end{aligned}
$$

com $\beta_{\dot{h}} \triangleq(1-\dot{h}(t)), \beta_{\dot{\tau}} \triangleq(1-\dot{\tau}(t))$. Usando (13)-(14) $\mathrm{e}$ (16) é possível obter

$$
\tilde{\Theta}(\alpha, t) \leq \Theta(\alpha)<\mathbf{0}
$$

assegurando, portanto, as condições do Lema 1. O fato de que $i i) \Rightarrow$ i) pode ser obtido através da transformação de congruência $\Theta(\alpha)=\mathcal{U}^{\prime} \Xi(\alpha) \mathcal{U}$ em que

$$
\mathcal{U} \triangleq\left[\begin{array}{ccc}
A(\alpha) & A_{h}(\alpha) & \mathbf{0} \\
\mathbf{I}_{n} & \mathbf{0} & -E(\alpha) \\
\hline & \mathbf{I}_{3 n} &
\end{array}\right]
$$

e, finalmente, $i i) \Leftarrow i$ ) pode ser provado utilizando o Lema de Finsler (de Oliveira e Skelton, 2001). De fato, escolhendo

$$
\omega=\left[\begin{array}{c}
\dot{\boldsymbol{\Delta}}\left(x_{\tau}\right) \\
\boldsymbol{\Delta}\left(x_{\tau}\right) \\
x(t) \\
x(t-h(t)) \\
x(t-\tau(t))
\end{array}\right]
$$

$\mathrm{e}$

$$
\begin{array}{r}
Q(\alpha)=\operatorname{diag}\left\{\left[\begin{array}{cc}
\mathbf{0} & P(\alpha) \\
P(\alpha) & \mathbf{0}
\end{array}\right], \quad S_{1}(\alpha)+S_{2}(\alpha),\right. \\
\left.-(1-\dot{h}(t)) S_{1}(\alpha), \quad-(1-\dot{\tau}(t)) S_{2}(\alpha)\right\}
\end{array}
$$

tem-se que que (12) e (1) podem ser escritas, respectivamente, como $\omega^{\prime} Q(\alpha) \omega<0$ e $\mathcal{B}(\alpha) \omega=\mathbf{0}$, com

$$
\mathcal{B}(\alpha)=\left[\begin{array}{ccccc}
\mathbf{I}_{n} & \mathbf{0} & -A(\alpha) & -A_{h}(\alpha) & \mathbf{0} \\
\mathbf{0} & \mathbf{I}_{n} & -\mathbf{I}_{n} & \mathbf{0} & E(\alpha)
\end{array}\right]
$$

Neste caso, o lema de Finsler assegura que essas condições são equivalentes à solução de

$$
Q(\alpha)+\mathcal{X}(\alpha) \mathcal{B}(\alpha)+\mathcal{B}(\alpha)^{\prime} \mathcal{X}(\alpha)^{\prime}<\mathbf{0}
$$

com

$$
\mathcal{X}(\alpha)=\left[\begin{array}{cc}
F_{1}(\alpha) & F_{2}(\alpha) \\
G_{1}(\alpha) & G_{2}(\alpha) \\
H_{1}(\alpha) & H_{2}(\alpha) \\
M_{1}(\alpha) & M_{2}(\alpha) \\
N_{1}(\alpha) & N_{2}(\alpha)
\end{array}\right]
$$




$$
\begin{aligned}
& \Xi(\alpha) \triangleq\left[\begin{array}{cc}
F_{1}(\alpha)+F_{1}(\alpha)^{\prime} & P(\alpha)+F_{2}(\alpha)+G_{1}(\alpha) \\
\star & G_{2}(\alpha)+G_{2}(\alpha)^{\prime} \\
\star & \star \\
\star & \star \\
\star & \star
\end{array}\right. \\
& M_{1}(\alpha)^{\prime}-F_{1}(\alpha) A_{h}(\alpha) \\
& M_{2}(\alpha)^{\prime}-G_{1}(\alpha) A_{h}(\alpha) \\
& -\left(H_{1}(\alpha) A_{h}(\alpha)\right. \\
& \left.+A(\alpha)^{\prime} M_{1}(\alpha)^{\prime}+M_{2}(\alpha)^{\prime}\right) \\
& -\beta_{\bar{h}} S_{1}(\alpha)-M_{1}(\alpha) A_{h}(\alpha) \\
& -A_{h}(\alpha)^{\prime} M_{1}(\alpha)^{\prime} \\
& H_{1}(\alpha)^{\prime}-\left(F_{1}(\alpha) A(\alpha)+F_{2}(\alpha)\right) \\
& H_{2}(\alpha)^{\prime}-\left(G_{1}(\alpha) A(\alpha)+G_{2}(\alpha)\right) \\
& S_{1}(\alpha)+S_{2}(\alpha)-\left(H_{1}(\alpha) A(\alpha)\right. \\
& \left.+A(\alpha)^{\prime} H_{1}(\alpha)^{\prime}+H_{2}(\alpha)+H_{2}(\alpha)^{\prime}\right) \\
& \star \\
& \star \\
& N_{1}(\alpha)^{\prime}+F_{2}(\alpha) E(\alpha) \\
& N_{2}(\alpha)^{\prime}+G_{2}(\alpha) E(\alpha) \\
& H_{2}(\alpha) E(\alpha)-\left(A(\alpha)^{\prime} N_{1}(\alpha)^{\prime}+N_{2}(\alpha)^{\prime}\right) \\
& <\mathbf{0} \\
& M_{2}(\alpha) E(\alpha)-A_{h}(\alpha)^{\prime} N_{1}(\alpha)^{\prime} \\
& -\beta_{\bar{\tau}} S_{2}(\alpha)+N_{2}(\alpha) E(\alpha)+E(\alpha)^{\prime} N_{2}(\alpha)^{\prime}
\end{aligned}
$$

A condição seguinte é convexa nas variáveis de otimização e de dimensão infinita em $\alpha$.

As condições apresentadas no Teorema 1 são convexas nas variáveis de decisão porém de dimensão infinita em $\alpha$. Além disso, não dependem dos valores de $\tau(t)$ e $h(t)$, mas apenas de suas taxas de variação máximas, respectivamente $\bar{\tau}$ e $\bar{h}$. O Teorema 1 poderia ser usado para verificar a estabilidade robusta de um sistema neutral pertencente a qualquer domínio de incertezas parametrizado em $\alpha$, ao preço de ser necessário testar todos os possíveis valores de $\alpha$ do domínio correspondente. Um teste numericamente eficiente, definido por um número finito de LMIs, que garante as condições do Lema 1, pode ser obtido impondose a seguinte estrutura às matrizes simétricas $P(\alpha), S_{1}(\alpha)$, $S_{2}(\alpha)$

$$
\begin{aligned}
P(\alpha) & =\sum_{j=1}^{N} \alpha_{j} P_{j} ; S_{1}(\alpha)=\sum_{j=1}^{N} \alpha_{j} S_{1 j} ; \\
S_{2}(\alpha) & =\sum_{j=1}^{N} \alpha_{j} S_{2 j} ; \sum_{j=1}^{N} \alpha_{j}=1, \alpha_{j} \geq 0
\end{aligned}
$$

e escolhendo as matrizes extras de forma independente do parâmetro $\alpha$, isto é

$$
\begin{aligned}
& F_{1}(\alpha)=F_{1}, \\
& F_{2}(\alpha)=F_{2}, \\
& G_{1}(\alpha)=G_{1}, \\
& G_{2}(\alpha)=G_{2},
\end{aligned}
$$

$$
\begin{aligned}
& H_{1}(\alpha)=H_{1}, \\
& H_{2}(\alpha)=H_{2}, \\
& M_{1}(\alpha)=M_{1}, \\
& M_{2}(\alpha)=M_{2}, \\
& N_{1}(\alpha)=N_{1} \mathrm{e} \\
& N_{2}(\alpha)=N_{2} .
\end{aligned}
$$

Note que estruturas mais complexas (por exemplo, com dependência afim no parâmetro $\alpha$ ) poderiam ser escolhidas para as matrizes extras que compõem o multiplicador $\mathcal{X}(\alpha)$ obtido pela aplicação do Lema de Finsler, implicando na existência de produtos de matrizes dependentes de parâmetros em (17). O produto de matrizes que são funções do parâmetro $\alpha$ poderia ser tratado utilizando a técnica apresentada em Ramos e Peres (2001), Ramos e Peres (2002), Leite e Peres (2003) e em Leite et al. (2004). Veja por exemplo (Peres et al., 2003) para a aplicação dessa técnica no contexto de sistemas incertos contínuos no tempo com atraso nos estados $(E=\mathbf{0})$, isto é, para os dois blocos do canto superior esquerdo de (16) $\operatorname{com} \beta_{\bar{h}}=1$ (atraso invariante no tempo).

Neste trabalho, optou-se pela escolha mais simples (matrizes extras fixas) que fornece resultados melhores do que os obtidos com a estabilidade quadrática a um custo computacional relativamente baixo e, além disso, permite recuperar condições existentes na literatura como casos particulares da condição mais geral aqui proposta por meio de transformações de congruência, como mostrado na próxima seção. 
Teorema 2 Se existirem matrizes simétricas definidas positivas $P_{j} \in \mathbb{R}^{n \times n}, S_{1 j} \in \mathbb{R}^{n \times n}, S_{2 j} \in \mathbb{R}^{n \times n}, j=$ $1, \ldots, N$ e matrizes $F_{1}, F_{2}, G_{1}, G_{2}, H_{1}, H_{2}, M_{1}, M_{2}, N_{1}$ e $N_{2}$ pertencentes a $\mathbb{R}^{n \times n}$ tais que

$$
\begin{aligned}
& \Xi_{j} \triangleq
\end{aligned}
$$

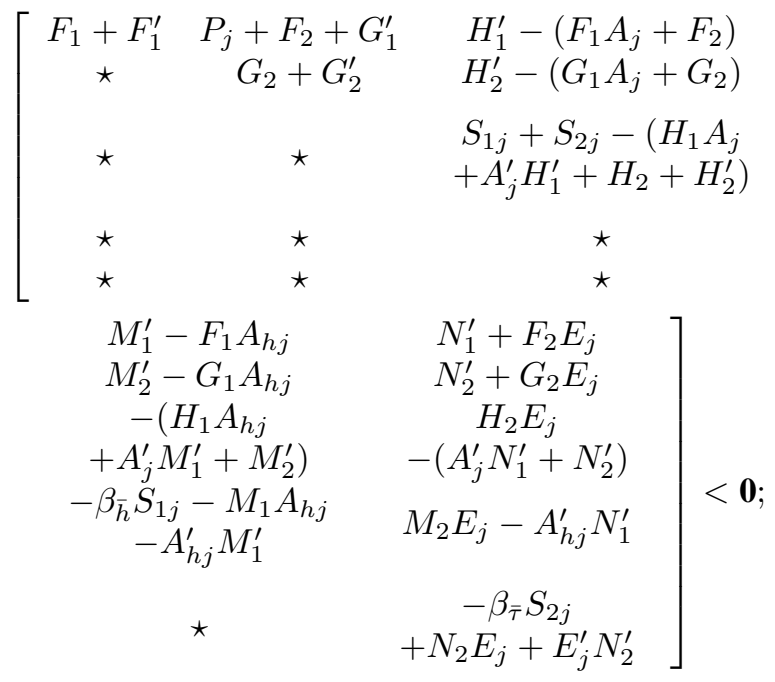

$$
\begin{aligned}
& j=1, \ldots, N
\end{aligned}
$$

então, as condições do Lema 1 são verificadas com $P(\alpha)$, $S_{1}(\alpha)$ e $S_{2}(\alpha)$ dadas por (18), assegurando, portanto, uma solução para o Problema 1.

Prova: Claramente, $P(\alpha), S_{1}(\alpha)$ e $S_{2}(\alpha)$ dadas em (18) $\operatorname{com} P_{j}=P_{j}^{\prime}>\mathbf{0}, S_{1 j}=S_{1 j}^{\prime}>\mathbf{0}, S_{2 j}=$ $S_{2 j}^{\prime}>\mathbf{0}, j=1, \ldots, N$ são matrizes definidas positivas. Multiplicando (19) por $\alpha_{j}, \alpha_{j} \geq 0, \sum_{j=1}^{N} \alpha_{j}=1$ e somando em $j=1, \ldots, N$, (17) é verificada.

É imediata a obtenção de uma condição baseada na estabilidade quadrática com variáveis extras a partir do Teorema 2. Esse resultado é apresentado no corolário que segue.

Corolário 1 Se existirem matrizes simétricas definidas positivas $P \in \mathbb{R}^{n \times n}, S_{1} \in \mathbb{R}^{n \times n}, S_{2} \in \mathbb{R}^{n \times n}$, e matrizes $F_{1}, F_{2}, G_{1}, G_{2}, H_{1}, H_{2}, M_{1}, M_{2}, N_{1}$ e $N_{2}$ pertencentes a $\mathbb{R}^{n \times n}$ tais que

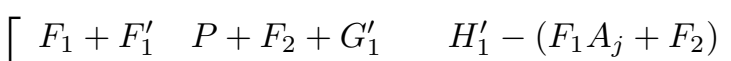

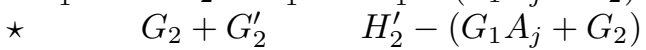

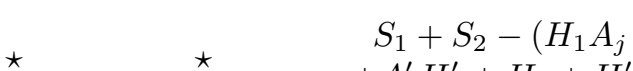

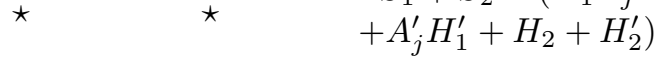

$$
\begin{aligned}
& \begin{array}{lll}
\star & \star & \star \\
\star & \star & \star
\end{array} \\
& M_{1}^{\prime}-F_{1} A_{h j} \quad N_{1}^{\prime}+F_{2} E_{j} \\
& M_{2}^{\prime}-G_{1} A_{h j} \quad N_{2}^{\prime}+G_{2} E_{j} \\
& -\left(H_{1} A_{h j} \quad H_{2} E_{j}\right. \\
& \left.+A_{j}^{\prime} M_{1}^{\prime}+M_{2}^{\prime}\right) \quad-\left(A_{j}^{\prime} N_{1}^{\prime}+N_{2}^{\prime}\right) \\
& \begin{array}{c}
-\beta_{\bar{h}} S_{1}-M_{1} A_{h j} \\
-A_{h j}^{\prime} M_{1}^{\prime}
\end{array} M_{2} E_{j}-A_{h j}^{\prime} N_{1}^{\prime} \\
& -\beta_{\bar{\tau}} S_{2} \\
& +N_{2} E_{j}+E_{j}^{\prime} N_{2}^{\prime} \\
& j=1, \ldots, N
\end{aligned}
$$

então, as condições do Lema 1 são verificadas $\operatorname{com} P(\alpha)=$ $P, S_{1}(\alpha)=S_{1}$ e $S_{2}(\alpha)=S_{2}$, assegurando, portanto, uma solução para o Problema 1. Neste caso o sistema incerto (1)(5) é dito "quadraticamente estável".

Note que a condição i) do Teorema 1 não pode ser diretamente transformada em uma condição de dimensão finita em $\alpha$ como feito com a condição ii) desse teorema. O principal obstáculo neste caso é a ocorrência do produto triplo nos blocos $(1,3)$ e $(2,3)$ (e correspondentes simétricos) de (16). Por essa razão, várias condições convexas encontradas na literatura exigem que a matriz $E(\alpha)$ seja precisamente conhecida, isto é $E(\alpha)=E, \forall \alpha$ (Bliman, 2002). No caso de $E(\alpha)=E$ pode-se obter uma condição de estabilidade quadrática para o sistema neutral com matrizes $A(\alpha)$ e $A_{h}(\alpha)$ incertas e atrasos $h(t)$ e $\tau(t)$ variantes no tempo e sujeitos a (13)-(14), conforme apresentado no seguinte corolário.

Corolário $2 \mathrm{Se}$ existirem matrizes simétricas definidas positivas $P \in \mathbb{R}^{n \times n}, S_{1} \in \mathbb{R}^{n \times n}$ e $S_{2} \in \mathbb{R}^{n \times n}$ tais que

$$
\left[\begin{array}{ccc}
A_{j}^{\prime} P+P A_{j}+S_{1}+S_{2} & P A_{h j} & -A_{j}^{\prime} P E \\
\star & -\beta_{\bar{h}} S_{1} & -A_{h j} P E \\
\star & \star & -\beta_{\bar{\tau}} S_{2}
\end{array}\right]<\mathbf{0}
$$

então, as condições do Lema 1 são verificadas $\operatorname{com} P(\alpha)=$ $P, S_{1}(\alpha)=S_{1}$ e $S_{2}(\alpha)=S_{2}$, garantindo que o sistema (1)(5), com $E_{j}=E, j=1, \ldots, N$ e atrasos sujeitos a (13) e (14), é quadraticamente estável. 


\section{OUTRAS CONDIÇÕES ESTABILIDADE ROBUSTA}

Alguns casos degenerados da equação diferencial hiperbólica dada em (1) são de interesse teórico e prático. Esses casos podem ser sumarizados pelas seguintes situações:

1. Matriz $A_{h}(\alpha)=\mathbf{0}, \forall \alpha$, implicando que o sistema (1) é descrito por

$$
\frac{\partial}{\partial t} \boldsymbol{\Delta}\left(x_{\tau}\right)=A(\alpha) x(t)
$$

2. Matriz $E(\alpha)=\mathbf{0}, \forall \alpha$, o que faz o sistema (1) deixar de ser do tipo neutral, descrito por uma equação diferencial hiperbólica, e recuperar o caso, mais conhecido, de um sistema incerto com atraso nos estados, dado por

$$
\dot{x}(t)=A(\alpha) x(t)+A_{h}(\alpha) x(t-h(t))
$$

Nesse caso, o operador $\boldsymbol{\Delta}(\cdot)$ em (2) é simplesmente $\boldsymbol{\Delta}\left(x_{\tau}\right)=x(t)$, pois, sem perda de generalidade, assume-se $\tau(t)=0, \forall t$.

3. As matrizes $E(\alpha)$ e $A_{h}(\alpha)$ são ambas nulas, $\forall \alpha$, recuperando o caso clássico de um sistema linear incerto contínuo no tempo e livre de atrasos dado por

$$
\dot{x}(t)=A(\alpha) x(t)
$$

Nessas três situações, condições de menor complexidade, em relação às já apresentadas neste trabalho, podem ser obtidas a partir das condições dos teoremas 1 e 2 para a análise de estabilidade robusta de (22)-(24). Esse fato decorre de simplificações no funcional de Lyapunov-Krasovskii (6), recuperando funcionais comumente empregados em cada uma dessas situações, conforme discutido a seguir. É bom lembrar que em (22)-(24) as matrizes dinâmicas pertencem ao politopo $\mathcal{P}$ dado em (5).

Note que para o caso em que $A_{h}(\alpha)=\mathbf{0}, \forall \alpha$, o funcional (6)-(9) pode ser simplificado impondo $S_{1}(\alpha)=\mathbf{0}$, resultando em

$$
\begin{aligned}
& \quad \begin{array}{l}
V(\alpha, x(t), h(t), \tau(t))=V(\alpha, x(t), \tau(t)) \\
=V_{1}\left(\alpha, \boldsymbol{\Delta}\left(x_{\tau}\right)\right)+V_{3}(\alpha, x(t), \tau(t))>0
\end{array}
\end{aligned}
$$

e, no caso em que apenas $E(\alpha)=\mathbf{0}, \forall \alpha$, toma-se $S_{2}(\alpha)=\mathbf{0}$ levando o funcional (6) à forma

$$
\begin{aligned}
& V(\alpha, x(t), h(t), \tau(t)) \\
& \quad=V_{1}\left(\alpha, \boldsymbol{\Delta}\left(x_{\tau}\right)\right)+V_{2}(\alpha, x(t), h(t))>0
\end{aligned}
$$

$$
\begin{aligned}
& V(\alpha, x(t), h(t), \tau(t))=V(\alpha, x(t), h(t)) \\
& =x(t)^{\prime} P(\alpha) x(t)+V_{2}(x(t), h(t))>0
\end{aligned}
$$

Na terceira situação, em que $A_{h}(\alpha)=\mathbf{0}$ e $E(\alpha)=\mathbf{0}, \forall \alpha$, o funcional em estudo fica reduzido ao funcional padrão de Lyapunov, sendo dado por

$$
\begin{aligned}
& V(\alpha, x(t), h(t), \tau(t)) \\
& \quad=V(\alpha, x(t))=x(t)^{\prime} P(\alpha) x(t)>0
\end{aligned}
$$

Na seqüência são apresentadas essas condições, que podem ser obtidas como casos particulares dos teoremas 1 e 2 . Neste trabalho é focalizada a obtenção de condições convexas de dimensão finita em $\alpha$ e por isso os corolários apresentados a seguir são determinados a partir das condições do Teorema 2. Um desenvolvimento análogo ao apresentado a seguir pode ser feito a partir do Teorema 1, obtendo, neste caso, condições de dimensão infinita em $\alpha$. É importante salientar que, embora essas condições possam ser obtidas a partir dos funcionais de Lyapunov-Krasovskii (25)-(28), seguindo passos semelhantes aos apresentados nas provas dos teoremas 1 e 2, optou-se pela obtenção dessas através da aplicação de transformações de congruência em (19).

\subsection{Caso $A_{h}(\alpha)=\mathbf{0}$}

A condição de estabilidade robusta independente do atraso para este caso pode ser obtida de (19), fazendo $S_{1 j}=\mathbf{0}$, $j=1, \ldots, N$, e aplicando a transformação de congruência

$$
T_{A_{h}} \Xi_{j} T_{A_{h}}^{\prime} ; j=1, \ldots, N
$$

$\operatorname{com} \Xi_{j}$ dado em (19) e

$$
T_{A_{h}}=\left[\begin{array}{ccccc}
\mathbf{I}_{n} & \mathbf{0} & \mathbf{0} & \mathbf{0} & \mathbf{0} \\
\mathbf{0} & \mathbf{I}_{n} & \mathbf{0} & \mathbf{0} & \mathbf{0} \\
\mathbf{0} & \mathbf{0} & \mathbf{I}_{n} & \mathbf{0} & \mathbf{0} \\
\mathbf{0} & \mathbf{0} & \mathbf{0} & \mathbf{0} & \mathbf{I}_{n}
\end{array}\right]
$$

que resulta no seguinte corolário.

Corolário $3 \mathrm{Se}$ existirem matrizes simétricas definidas positivas $P_{j} \in \mathbb{R}^{n \times n}, S_{2 j} \in \mathbb{R}^{n \times n}, j=1, \ldots, N$ e matrizes $F_{1}, F_{2}, G_{1}, G_{2}, H_{1}, H_{2}, N_{1}$ e $N_{2}$ pertencentes a $\mathbb{R}^{n \times n}$ tais 
que

$$
\begin{gathered}
\Xi_{A_{h} j} \triangleq\left[\begin{array}{cc}
F_{1}+F_{1}^{\prime} & P_{j}+F_{2}+G_{1}^{\prime} \\
\star & G_{2}+G_{2}^{\prime} \\
\star & \star \\
\star & \star \\
H_{1}^{\prime}-\left(F_{1} A_{j}+F_{2}\right) & N_{1}^{\prime}+F_{2} E_{j} \\
H_{2}^{\prime}-\left(G_{1} A_{j}+G_{2}\right) & N_{2}^{\prime}+G_{2} E_{j} \\
S_{2 j}-\left(H_{1} A_{j}\right. & H_{2} E_{j} \\
\left.+A_{j}^{\prime} H_{1}^{\prime}+H_{2}+H_{2}^{\prime}\right) & -\left(A_{j}^{\prime} N_{1}^{\prime}+N_{2}^{\prime}\right) \\
\star & N_{2} E_{j}+E_{j}^{\prime} N_{2}^{\prime} \\
& -\beta_{\bar{\tau}} S_{2 j}
\end{array}\right]<\mathbf{0} ; \\
\quad j=1, \ldots, N
\end{gathered}
$$

então, $P(\alpha)$ e $S_{2}(\alpha)$ dadas em (18) asseguram (25) e (11), garantindo a estabilidade robusta de (22) com $\tau(t)$ sujeito a (14), independentemente do valor do atraso $\tau(t)$.

\subsection{Caso $E(\alpha)=\mathbf{0}$}

Neste caso a característica "neutral" do sistema (1) é perdida, passando este a ser descrito como em (23), com $h(t)$ sujeito a (13). Fazendo $S_{2 j}=\mathbf{0}$ em (19) e aplicando a transformação de congruência

$$
\Xi_{E j}=T_{E} \Xi_{j} T_{E}^{\prime} ; j=1, \ldots, N
$$

com $\Xi_{j}$ dado em (19) e

$$
T_{E}=\left[\begin{array}{ccccc}
\mathbf{I}_{n} & \mathbf{0} & \mathbf{0} & \mathbf{0} & \mathbf{0} \\
\mathbf{0} & \mathbf{I}_{n} & \mathbf{I}_{n} & \mathbf{0} & \mathbf{0} \\
\mathbf{0} & \mathbf{0} & \mathbf{0} & \mathbf{I}_{n} & \mathbf{0}
\end{array}\right]
$$

pode-se estabelecer o seguinte corolário.

Corolário 4 Se existirem matrizes positivas definidas $P_{j} \in$ $\mathbb{R}^{n \times n}$ e $S_{1 j} \in \mathbb{R}^{n \times n}, j=1, \ldots, N$, matrizes $F \in \mathbb{R}^{n \times n}$, $G \in \mathbb{R}^{n \times n}, M \in \mathbb{R}^{n \times n}$ tais que

$$
\begin{aligned}
\Xi_{E j} \triangleq & {\left[\begin{array}{cc}
F+F^{\prime} & P_{j}+G^{\prime}-F A_{j} \\
\star & S_{1 j}-G A_{j}-A_{j}^{\prime} G^{\prime} \\
\star & \star \\
& M^{\prime}-F A_{h j} \\
-G A_{h j}-A_{j}^{\prime} M^{\prime} \\
-\beta_{\bar{h}} S_{1 j}-M A_{h j}-A_{h j}^{\prime} M^{\prime}
\end{array}\right]<\mathbf{0} ; } \\
& \quad j=1, \ldots, N
\end{aligned}
$$

então, $P(\alpha)$ e $S_{1}(\alpha)$ dadas em (18) asseguram (27) e (11), garantindo a estabilidade robusta de (23) com $h(t)$ sujeito a (13), independentemente do valor do atraso $h(t)$.

No Corolário 4, as variáveis extras $F, G$ e $M$ estão relacionadas às do Teorema 2 da seguinte forma:

$$
F=F_{1} ; \quad G=G_{1}+H_{1} ; \text { e } M=M_{1}
$$

\subsection{Caso $E(\alpha)=\mathbf{0}$ e $A_{h}(\alpha)=\mathbf{0}$}

Finalmente, considere o sistema linear incerto dado em (1) livre de atrasos. Portanto, o sistema contínuo no tempo resultante é dado por

$$
\dot{x}(t)=A(\alpha) x(t)
$$

Neste caso, a condição apresentada por Peaucelle et al. (2000) para a estabilidade robusta de sistemas contínuos no tempo pode ser recuperada a partir de (19) impondo $S_{1 j}=$ $S_{2 j}=\mathbf{0}, j=1, \ldots, N$ e considerando, portanto, o funcional dado em (28). Aplicando a transformação de congruência

$$
T_{E A_{h}} \Xi_{j} T_{E A_{h}}^{\prime} ; j=1, \ldots, N
$$

com $\Xi_{j}, j=1, \ldots, N$, dado em (19) e

$$
T_{E A_{h}}=\left[\begin{array}{ccccc}
\mathbf{I}_{n} & \mathbf{0} & \mathbf{0} & \mathbf{0} & \mathbf{0} \\
\mathbf{0} & \mathbf{I}_{n} & \mathbf{I}_{n} & \mathbf{0} & \mathbf{0}
\end{array}\right]
$$

o seguinte corolário é obtido.

Corolário $5 \mathrm{Se}$ existirem matrizes simétricas definidas positivas $P_{j} \in \mathbb{R}^{n \times n}, j=1, \ldots, N$, matrizes $F \in \mathbb{R}^{n \times n}$ e $G \in \mathbb{R}^{n \times n}$ tais que

$$
\begin{array}{cc}
\Xi_{E A_{h} j} \triangleq\left[\begin{array}{cc}
-\left(F A_{j}+A_{j}^{\prime} F^{\prime}\right) & P_{j}+F-A_{j}^{\prime} G \\
\star & G+G^{\prime}
\end{array}\right]<\mathbf{0} \\
j=1, \ldots, N
\end{array}
$$

então, $P(\alpha)$ dada em (18) assegura (28) e (11), garantindo a estabilidade robusta do sistema livre de atrasos dado por (24).

No caso do Corolário 5 as relações entre as matrizes $F$ e $G$ em (33), as correspondentes em Peaucelle et al. (2000) $\left(F_{\mathrm{P}}\right.$, $\left.G_{\mathrm{P}}\right)$ e as matrizes extras de (19) são dadas por

$$
G=G_{\mathrm{P}}=-\left(G_{1}+H_{1}\right) ; \quad F=F_{\mathrm{P}}=-F_{1}
$$

Observação 1 É importante salientar que todos os resultados apresentados aqui contêm, como caso particular, a estabilidade quadrática que utiliza matrizes constantes nos funcionais de Lyapunov-Krasovskii considerados. Essas condições podem ser obtidas dos resultados aqui apresentados impondo-se $P(\alpha)=P_{j}=P$, $S_{1}(\alpha)=S_{1 j}=S_{1}$ e $S_{2}(\alpha)=S_{2 j}=S_{2}, j=1, \ldots, N$.

Observação 2 A extensão dos resultados apresentados neste trabalho para o caso de múltiplos atrasos é imediata. Nesse caso, utiliza-se uma matriz $S_{1}(\alpha)_{\kappa}$ para cada atraso $h_{\kappa}(t), \kappa=1, \ldots, \bar{\kappa}$, e uma matriz $S_{2}(\alpha)_{\mu}$ para cada atraso $\tau_{\mu}(t), \mu=1, \ldots, \bar{\mu}$. Para detalhes desse tipo de extensão, veja (Niculescu, 2001). 


\section{COMPLEXIDADE NUMÉRICA}

A complexidade numérica associada às condições LMI apresentadas neste trabalho são dadas na Tabela 1. Essas LMIs podem ser resolvidas em tempo polinomial através de algoritmos com complexidade numérica proporcional a $\mathcal{K}^{3} \mathcal{L}$, sendo $\mathcal{K}$ o número de variáveis escalares e $\mathcal{L}$ o número de linhsa das LMIs (Gahinet et al., 1995).

Tabela 1: Número de variáveis escalares $(\mathcal{K})$ e número de linhas $(\mathcal{L})$ em função do número de estados $(n)$ e vértices $(N)$ para as condições de análise de estabilidade estudadas.

\begin{tabular}{|c|c|c|}
\hline \hline Critério & $\mathcal{K}(\mathrm{n}-$ de variáveis $)$ & $\mathcal{L}(\mathrm{n}-$ de linhas $)$ \\
\hline \hline Teorema 2 & $\frac{3 N n}{2}(n+1)+10 n^{2}$ & $8 N n$ \\
\hline Corolário 1 & $\frac{3 N n}{2}(n+1)+10 n^{2}$ & $8 N n$ \\
\hline Corolário 2 & $\frac{3 n}{2}(n+1)$ & $3 N n$ \\
\hline Corolário 3 & $N n(n+1)+8 n^{2}$ & $6 N n$ \\
\hline Corolário 4 & $N n(n+1)+3 n^{2}$ & $5 N n$ \\
\hline Corloário 5 & $\frac{N n}{2}(n+1)+2 n^{2}$ & $3 N n$ \\
\hline \hline
\end{tabular}

Uma outra abordagem, menos conservadora, para obtenção de condições convexas de dimensão finita suficientes para aquelas estabelecidas no Teorema 1 é utilizar as idéias já exploradas em Ramos e Peres (2001), Ramos e Peres (2002), Leite e Peres (2003) e em Leite et al. (2004), permitindo que as variáveis extras presentes na condição $i i)$ desse teorema sejam também dependentes, de forma afim, do parâmetro $\alpha$. É claro que, nesse caso, as condições obtidas serão menos conservadoras que as condições do Teorema 2, porém com complexidade mais elevada. Como exemplo desse tipo de abordagem, no contexto de sistemas incertos contínuos no tempo com atrasos (invariantes no tempo) nos estados, veja (Leite et al., 2003) para condições independentes do atraso com variáveis extras e (Peres et al., 2003) para condições dependentes e independentes do atraso (sem variáveis extras).

\section{EXEMPLOS}

Exemplo 1 Considere o sistema linear incerto neutral com dois estados pertencente a um domínio politópico definido por três vértices $V_{j} \equiv\left(E, A, A_{h}\right)_{j}, j=1,2,3$, em que

$$
E_{1}=-0.10 \mathbf{I}_{2} ; \quad E_{2}=-0.15 \mathbf{I}_{2} ; \quad E_{3}=-0.20 \mathbf{I}_{2}
$$

$$
\begin{aligned}
& A_{1}=\left[\begin{array}{cc}
-0.7085 & -0.0758 \\
-0.2511 & -0.0269
\end{array}\right] \\
& A_{2}=\left[\begin{array}{cc}
-0.3260 & 0.2616 \\
0.2980 & -0.2393
\end{array}\right] ; \\
& A_{3}=\left[\begin{array}{cc}
-0.3957 & 0.3236 \\
0.3513 & -0.2874
\end{array}\right] \\
& A_{h 1}=\left[\begin{array}{cc}
-0.3543 & -0.0379 \\
-0.1256 & -0.0135
\end{array}\right] \\
& A_{h 2}=\left[\begin{array}{cc}
-0.1630 & 0.1308 \\
0.1490 & -0.1196
\end{array}\right] \\
& A_{h 3}=\left[\begin{array}{cc}
-0.1978 & 0.1618 \\
0.1756 & -0.1437
\end{array}\right]
\end{aligned}
$$

Usando os resultados do Teorema 2, é possível caracterizar a região $(\bar{h}, \bar{\tau})$ na qual este sistema é robustamente estável independentemente dos valores dos atrasos $h(t)$ e $\tau(t)$, como mostrado na Figura 1, em que foi usada uma malha de 0.02 nos eixos $\bar{\tau}$ e $\bar{h}$. Salienta-se que esse sistema não pode ser caracterizado como quadraticatimente estável, isto é, as condições do Teorema 2 não são factíveis quando é imposto $P_{j}=P, S_{1 j}=S_{1}$ e $S_{2 j}=S_{2}, j=1, \ldots, N$.

Exemplo 2 Considere o sistema linear incerto com atraso nos estados, cuja equação dinâmica é dada em (23), com $A(\alpha)$ e $A_{h}(\alpha)$ pertencentes ao politopo determinado pelos vértices $V_{1}=\left[\begin{array}{ll}A_{1}, & A_{h 1}\end{array}\right], V_{2}=\left[\begin{array}{ll}A_{2}, & A_{h 2}\end{array}\right]$ e $V_{3}=$ $\left[A_{3}, A_{h 3}\right]$ em que

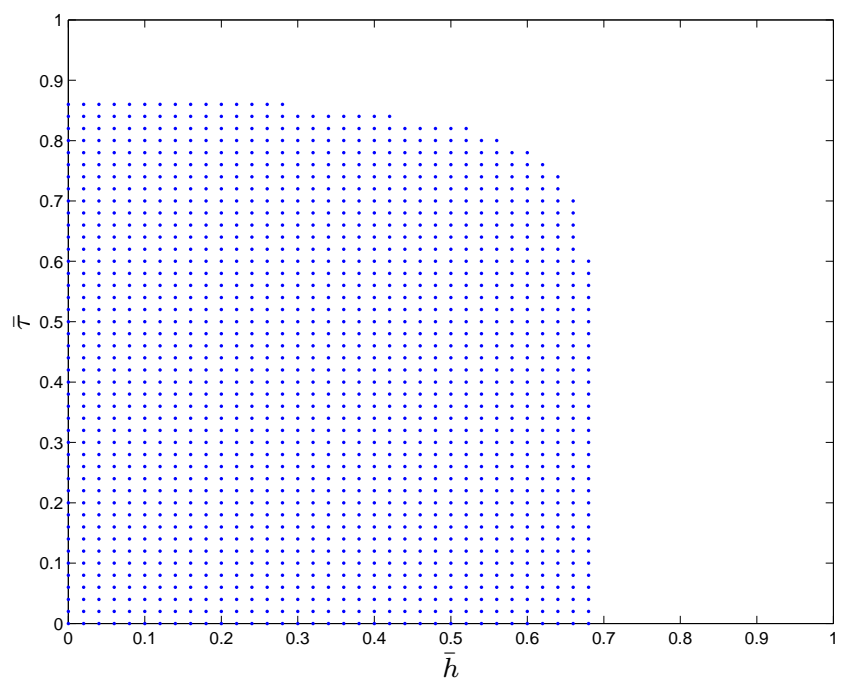

Figura 1: Região $(\bar{h}, \bar{\tau})$ na qual é verificada a estabilidade robusta do sistema linear incerto neutral dado por (34)-(40), obtida através das condições do Teorema 2. 


$$
\begin{aligned}
& A_{1}=\left[\begin{array}{cc}
-0.8550 & 0.4346 \\
0.5832 & -0.2965
\end{array}\right] \\
& A_{2}=\left[\begin{array}{cc}
-0.8080 & 0.1023 \\
0.5095 & -0.0646
\end{array}\right] \\
& A_{3}=\left[\begin{array}{cc}
-0.9438 & -0.0786 \\
0.5716 & 0.0476
\end{array}\right] \\
& A_{h 1}=\left[\begin{array}{cc}
-0.4275 & 0.2173 \\
0.2916 & -0.1482
\end{array}\right] \\
& A_{h 2}=\left[\begin{array}{cc}
-0.4040 & 0.0511 \\
0.2548 & -0.0323
\end{array}\right] \\
& A_{h 3}=\left[\begin{array}{cc}
-0.4719 & -0.0393 \\
0.2858 & 0.0238
\end{array}\right]
\end{aligned}
$$

Esse sistema não é quadraticamente estável (independentemente do valor do atraso $h(t)$ ). Utilizando as condições LMI estabelecidas no Teorema 2 , com $E_{j}=\mathbf{0}$ e $\beta_{\bar{\tau}}=1$, é verificado que esse sistema é robustamente estável independentemente do atraso $h(t)$ para $|\dot{h}(t)| \leq \bar{h}=0.4047$. O mesmo resultado é obtido pelas condições do Corolário 4. $\mathrm{Na}$ verdade, neste caso (i.e. $E_{j}=\mathbf{0}$ e $\beta_{\bar{\tau}}=1$ ), as condições do Corolário 4 são equivalentes às condições do Teorema 2, isto é, se as condições do Corolário 4 forem verificadas, então existem $S_{2 j}, j=1, \ldots, N$ que verificam as condições do Teorema 2 para o mesmo valor de $\beta_{\bar{\tau}}$. Reciprocamente, se $\Xi_{j}<0, j=1, \ldots, N$, então a transformação de congruência dada por (31) garante que as condições do Corolário 4 também valem para qualquer valor de $S_{2 j}>0$, $j=1, \ldots, N$.

\section{CONCLUSÕES}

Condições LMI independentes do atraso, suficientes para a análise de estabilidade robusta de sistemas lineares incertos neutrais, pertencentes a domínios politópicos, com atrasos variantes no tempo, foram apresentadas. Essas condições são baseadas em funcionais de LyapunovKrasovskii dependentes de parâmetro, o que torna as condições bem menos conservadoras que outras baseadas em matrizes fixas (estabilidade quadrática). Foi mostrado que as condições de análise de estabilidade robusta de sistemas neutrais contêm outras condições específicas para casos degenerados, tais como sistemas com atrasos e sistemas livres de atraso. Exemplos numéricos ilustram os resultados.

\section{AGRADECIMENTOS}

Este trabalho contou com o apoio das agências CAPES, CNPq, FAPEMIG (TEC 840/05) e FAPESP. Os autores agradecem os comentários e as sugestões dos revisores anônimos.

\section{REFERÊNCIAS}

Barmish, B. R. (1985). Necessary and sufficient conditions for quadratic stabilizability of an uncertain system, Journal of Optimization Theory and Applications 46(4): 399-408.

Bellen, A., Guglielmi, N. e Ruehli, A. E. (1999). Methods for linear systems of circuit delay differential equations of neutral type, IEEE Transactions on Circuits and Systems Part I: Fundamental Theory and Applications 46(1): 212-216.

Bin, Y., Ruijun, Z. e Tao, L. (2003). Delay-dependent stability criterion for a class of neutral time-delay systems, Proceedings of the 2003 American Control Conference, Denver, CO, pp. 2694-2696.

Bliman, P.-A. (2002). Lyapunov equation for the stability of linear delay systems of retarded and neutral type, IEEE Transactions on Automatic Control 47(2): 327-335.

Cao, D. Q. e He, P. (2004). Stability criteria of linear neutral systems with a single delay, Applied Mathematics and Computation 148(1): 135-143.

Chen, J.-D. (2003). New stability criteria for a class of neutral systems with discrete and distributed timedelays: an LMI approach, Applied Mathematics and Computation 150(3): 719-736.

Chen, J.-D. (2004). Robust control for uncertain neutral systems with time-delays in state and control input via LMI and GAs, Applied Mathematics and Computation 157(2): 535-548.

Cullum, J., Ruehli, A. e Zhang, T. (2000). A method for reduced-order modeling and simulation of large interconnect circuits and its application to PEED models with retardation, IEEE Transactions on Circuits and Systems Part II: Analog and Digital Signal Processing 47(4): 261-273.

de Oliveira, M. C., Bernussou, J. e Geromel, J. C. (1999). A new discrete-time robust stability condition, Systems \& Control Letters 37(4): 261-265.

de Oliveira, M. C. e Skelton, R. E. (2001). Stability tests for constrained linear systems, in S. O. Reza Moheimani (ed.), Perspectives in Robust Control, Vol. 268 of Lecture Notes in Control and Information Science, Springer-Verlag, New York, pp. 241-257.

Dugard, L. e Verriest, E. I. (1997). Stability and Control of Time-delay Systems, Springer-Verlag, Berlin, Germany.

El'sgol'ts, L. E. (1966). Introduction to the theory of differential equations with deviating arguments, Holden-Day, Inc., San Francisco, USA. 
Fridman, E. (2001). New Lyapunov-Krasovskii functionals for stability of linear retarded and neutral type systems, Systems \& Control Letters 43(4): 309-319.

Fu, X. (2004). Controllability of abstract neutral functional differential systems with unbounded delay, Applied Mathematics and Computation 151(2): 299-314.

Gahinet, P., Nemirovskii, A., Laub, A. J. e Chilali, M. (1995). LMI Control Toolbox User's Guide, The Math Works Inc., Natick, MA.

Gu, K., Kharitonov, V. L. e Chen, J. (2003). Stability of Time-delay Systems, Control Engineering, Birkhäuser, Boston, MA.

Gu, K. e Niculescu, S. (2003). Survey on recent results in the stability and control of time-delay systems, Journal of Dynamic Systems, Measurement and Control Transactions of ASME 125: 125-165.

Gu, K. e Niculescu, S.-I. (2001). Further remarks on additional dynamics in various model transformations of linear delay systems, IEEE Transactions on Automatic Control 46(3): 497-500.

Hale, J. (1977). Theory of Functional Differential Equations, Springer-Verlag, New York.

Hale, J. e Lunel, S. M. V. (1993). Introduction to Functional Differential Equations, Springer-Verlag, New York.

Han, Q.-L. (2002). Robust stability of uncertain delaydifferential systems of neutral type, Automatica 38(4): 719-723.

Han, Q.-L. (2004). A descriptor system approach to robust stability of uncertain neutral system with discrete and distributed delays, Automatica 40(10): 1791-1796.

Ivănescu, D., Niculescu, S.-I., Dugard, L., Dion, J.-M. e Verriest, E. I. (2003). On delay-dependent stability for linear neutral systems, Automatica 39(2): 255-261.

Kao, C.-Y. e Rantzer, A. (2003). Stability criteria for systems with bounded uncertain time-varying delay, Proceedings of the 2003 European Control Conference, Cambridge, UK.

Kolmanovskii, V. B., Niculescu, S. I. e Richard, J. P. (1999). On the Liapunov-Krasovskii functionals for stability analysis of linear delay systems, International Journal of Control 72(4): 374-384.

Kolmanovskii, V. B. e Richard, J. P. (1999). Stability of some linear systems with delays, IEEE Transactions on Automatic Control 44(5): 984-989.
Kolmanovskii, V. e Myshkis, A. (1992). Applied Theory of Functional Differential Equations, Kluwer Academic Publishers, Dordrecht.

Kolmanovskii, V. e Myshkis, A. (1998). Introduction to the Theory and Applications of Functional Differential Equations, Kluwer Academic Publishers, Dordrecht.

Leite, V. J. S., Montagner, V. F., de Oliveira, P. J., Oliveira, R. C. L. F., Ramos, D. C. W. e Peres, P. L. D. (2004). Estabilidade robusta de sistemas lineares através de desigualdades matriciais lineares, SBA Controle \& Automação 15(1): 24-40.

Leite, V. J. S. e Peres, P. L. D. (2003). An improved LMI condition for robust $\mathcal{D}$-stability of uncertain polytopic systems, IEEE Transactions on Automatic Control 48(3): 500-504.

Leite, V. J. S., Peres, P. L. D. e Tarbouriech, S. (2003). Less conservative time-delay independent LMI conditions for continuous-time polytopic systems, Proceedings of the 4th IFAC International Workshop on Linear Time Delay Systems, Rocquencourt, France. in CD-rom.

Mahmoud, M. S. (2000). Robust Control and Filtering for Time-Delay Systems, Control Engineering Series, Marcel Dekker, Inc., New York.

Malek-Zavarei, M. e Jamshidi, M. (1987). Time-Delay Systems: Analysis, Optimization and Applications, North-Holland, Amsterdam, The Netherlands.

Niculescu, S.-I. (2001). Delay Effects on Stability: A Robust Control Approach, Vol. 269 of Lecture Notes in Control and Information Sciences, Springer-Verlag, London.

Park, J.-H. (2001). A new delay-dependent criterion for neutral systems with multiple delays, Journal of Computational and Applied Mathematics 136(12): $177-184$

Park, J. H. (2003). Simple criterion fot asymptotic stability of interval neutral delay-differential systems, Applied Mathematics Letters 16(7): 1063-1068.

Park, J. H., Kwon, O. e Won, S. (2004). LMI approach to robust $\mathcal{H}_{\infty}$ filtering for neutral delay differential systems, Applied Mathematics and Computation 150(1): 235-244.

Park, J. H. e Won, S. (2000). Stability analysis for neutral delay-differential systems, Journal of The Franklin Institute 337(1): 1-9.

Peaucelle, D., Arzelier, D., Bachelier, O. e Bernussou, J. (2000). A new robust $\mathcal{D}$-stability condition for real convex polytopic uncertainty, Systems \& Control Letters 40(1): 21-30. 
Peres, P. L. D., Tarbouriech, S., Garcia, G. e Leite, V. J. S. (2003). Robust stability of time-delay continuous-time systems in polytopic domains, Proceedings of the 2003 European Control Conference, Cambridge, UK. in CDrom.

Ramos, D. C. W. e Peres, P. L. D. (2001). A less conservative LMI condition for the robust stability of discretetime uncertain systems, Systems \& Control Letters 43(5): 371-378.

Ramos, D. C. W. e Peres, P. L. D. (2002). An LMI condition for the robust stability of uncertain continuous-time linear systems, IEEE Transactions on Automatic Control 47(4): 675-678.

Richard, J.-P. (2003). Time-delay systems: an overview of some recent advances and open problems, Automatica 39(10): 1667-1694.

Skorodinskii, V. I. (1990). Iterational method of construction of Lyapunov-Krasovskii functionals for linear systems with delay, Automation and Remote Control 51(9): 1205-1212.

Verriest, E. I. (1999). Robust stability and adaptive control of time-varying neutral systems, Proceedings of the 38th IEEE Conference on Decision and Control, Phoenix, AZ, pp. 4690-4695.

Vieira, A. e Kailath, T. (1977). On another approach to the Schur-Cohn criterion, IEEE Transactions on Circuits and Systems CAS-24: 218-220.

Xu, S., Lam, J., Yang, C. e Verriest, E. I. (2003). An LMI approach to guaranteed cost control for uncertain linear neutral delay systems, International Journal of Robust and Nonlinear Control 13(1): 35-53.

Yue, D. e Han, Q.-L. (2004). A delay-dependent stability criterion of neutral systems and its application to a partial element equivalent circuit model, IEEE Transactions on Circuits and Systems Part II: Analog and Digital Signal Processing 51(12): 685-689.

Zhang, J., Knospe, C. e Tsiotras, P. (2001). Stability of time-delay systems: equivalence between Lyapunov and scaled small-gain conditions, IEEE Transactions on Automatic Control 46(3): 482-486. 